\title{
Switchable Dual-Wavelength Cylindrical Vector Beam Generation From a Passively Mode-Locked Fiber Laser Based on Carbon Nanotubes
}

\author{
Zuxing Zhang ${ }^{(\mathbb{1}}$, Yu Cai, Jie Wang, Hongdan Wan ${ }^{(0)}$, and Lin Zhang
}

\begin{abstract}
Cylindrical vector beams (CVBs) with axial symmetry in both polarization and field intensity have attracted much attention because of their unique optical properties. Conventional methods to obtain CVBs including direct modulation of light beams in free space and high-order mode excitation by offset splicing single-mode fiber with few-mode fiber usually works at single wavelength with rather narrow bandwidth. Here, for the first time to the best of our knowledge, we demonstrate switchable dual-wavelength CVB generation from a passively mode-locked fiber laser using carbon nanotubes as saturable absorber for mode-locking and a home-made mode-selective coupler as both mode converter and birefringence filter. In experiments, the mode-locked fiber laser delivers CVB pulses of dual-wavelength $(1532.5 \mathrm{~nm}$ and $1555.5 \mathrm{~nm})$ and corresponding single wavelength with duration of hundreds of femtosecond, respectively. Moreover, the output polarization status is switchable between radially and azimuthally polarized states. The mode-locked CVBs with wavelength and polarization flexibility may have potential applications in mode-division multiplexing optical fiber communication, nanoparticle manipulation, material processing, nonlinear optics, and so on.
\end{abstract}

Index Terms-Dual-wavelength, cylindrical vector beam, modelocked fiber lasers, mode-selective coupler, carbon nanotubes.

\section{INTRODUCTION}

I N RECENT years, with the development of communication technology, the human society has entered an unprecedented

Manuscript received July 30, 2017; revised September 28, 2017; accepted September 30, 2017. Date of publication October 11, 2017; date of current version November 1, 2017. This work was supported in part by the National Science Foundation of Jiangsu Province under Grants BK20161521 and BK20150858, in part by the Nanjing University of Posts and Telecommunications under Grants NY214059, NY214002, and NY215002, in part by the Distinguished Professor Project of Jiangsu under Grant RK002STP14001, in part by the Six Talent Peaks Project in Jiangsu Province under Grant 2015-XCL-023, and in part by the Postgraduate Research and Practice Innovation Program of Jiangsu Province under Grants SJCX17_0234 and KYCX17_0744. (Corresponding authors: Zuxing Zhang; Hongdan Wan.)

Z. Zhang is with the Advanced Photonic Technology Lab, Nanjing University of Posts and Telecommunications, Nanjing 210023, China, and also with the Aston Institute of Photonic Technologies, Aston University, Birmingham B4 7ET, U.K. (e-mail: zxzhang@njupt.edu.cn).

Y. Cai, J. Wang, and H. Wan are with the Advanced Photonic Technology Lab, Nanjing University of Posts and Telecommunications, Nanjing 210023, China (e-mail:1103725396@qq.com; 742413406@qq.com; hdwan@njupt.edu.cn).

L. Zhang is with the Advanced Photonic Technology Lab, Nanjing University of Posts and Telecommunications, Nanjing 210023, China, and also with the Aston Institute of Photonic Technologies, Aston University, Birmingham B4 7ET, U.K. (e-mail: 1.zhang@aston.ac.uk).

Color versions of one or more of the $f$ gures in this paper are available online at http://ieeexplore.ieee.org.

Digital Object Identifie 10.1109/JSTQE.2017.2761126 period of rapid growth of information. With successively using wavelength-division multiplexing (WDM), polarization multiplexing, coherent receiver and multi-order modulation technology, for light in fibe all degrees of freedom: frequency, polarization, amplitude and phase have been used to increase the capacity of the transmission system. However, the system capacity is still approaching the Shannon limit [1]-[3]. In order to further improve the capacity of the optical fibe transmission system and solve the bottleneck problem of increasingly tight bandwidth resource, the mode-division multiplexing technology based on few-mode or multi-mode fiber has become an promising scheme [4]. Therefore, it is critical to investigate fibe lasers with high-order mode emission, especially multiwavelength high-order mode generation.

CVB is a kind of non-uniform polarized beam with axial symmetry on the cross section of electric fiel [5], including radially polarization, azimuthally polarization and hybrid polarization. Due to its unique characteristics, it has been utilized in many ways, such as particle capture [6], high-resolution measurement [7], material processing [8] and electronic acceleration [9]. The intracavity or extracavity involvement of devices with axial birefringence, dichroism or spatially variant polarization property [10], [11] to render the necessary mode discrimination against the fundamental mode, forces the lasers to oscillate in CVB modes or converts spatially homogeneous polarizations into spatially inhomogeneous CVB polarizations. However, the use of bulky devices or complex fabrication process of such devices seems an obstacle to develop compact and robust CVB fibe lasers. As few-mode step-index optical fibe can support both azimuthally polarized TE01 and radially polarized TM01 modes, CVB generation with few-mode fibe based components with a function of transverse mode selection is a desirable scheme [12], [13]. Through lateral offset splicing two-mode fibe (TMF) with single-mode fibe (SMF) to excite high-order modes and utilizing a fibe Bragg grating (FBG) written on TMF to discriminate LP01 and LP11 modes [14], continuous [15][17], Q-switched [18], [19], and mode-locked [20]-[22] CVB fibe lasers have been implemented. However, due to mode fiel mismatch, offset splicing inevitably induces excess loss, and the FBG presents a rather narrow bandwidth for mode conversion.

In terms of fibe-based broadband mode converter, Witkowska et al. [23] presented two mode converters based on photonic crystal fiber (PCFs) by controlling hole collapse in PCF or using the ferrule technique for joining conventional 


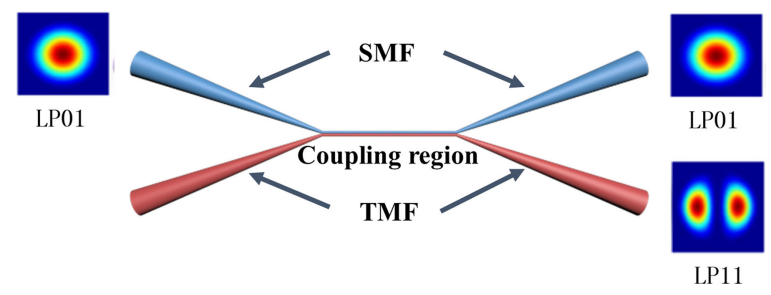

Fig. 1. The schematic of the MSC.

single-mode fiber to PCFs without splicing, with which mode conversion with high extinction ratio has been realized. However, the used fabrication process was relatively complicated. Based on the combination of a few-mode fibe and a SMF, Ismaeel et al. [24] demonstrated a MSC with relatively simple configuratio and fabrication process, which can excite specifi higher-order modes with high efficien y and purity. Recently, femtosecond [25] CVB pulse generation from modelocked fibe laser based on nonlinear polarization rotation effect and nanosecond [26] CVB pulse generation from mode-locked fibe laser based on nonlinear amplifie loop mirror have been reported based on the MSCs with SMF and TMF. Therefore, MSC composed of both SMF and few-mode fibe can convert the fundamental mode in SMF to the higher-order modes in few-mode fibe with high conversion efficien y and broad conversion bandwidth. Regarding mode-locking methods, carbon nanotubes (CNTs) and graphene have emerged as promising saturable absorbers [27]-[29] with ultrafast recovery time [30], [31], able to support short pulses [32], [33], and with a number of favorable properties for laser development, such as broadband operation [34], and integration into all-fibe configuration [35], [36]. However, there are few works about multiwavelength mode-locked CVB fibe laser.

Here, we propose and demonstrate a switchable dualwavelength passively mode-locked CVB fibe laser for the firs time, to the best of our knowledge. Compared with the method of using FM-FBG to generate CVB, the MSC has a smaller insertion loss and can work in broader conversion bandwidth. The CNTs-based saturable absorber is used to mode-lock the fibe laser, while the MSC converts the fundamental mode to the high-order mode and facilitates birefringence filtering In result, the CVB fibe laser can operate and switch at two different wavelengths through adjusting the orientations of the intracavity polarization controller (PC), due to the birefringence filterin effect introduced by the MSC. Both radially and azimuthally polarized beams can be obtained from the fibe laser. This all-fibe -based CVB pulse laser can provide a cost-effective multiwavelength CVB source for practical applications.

\section{Mode-Selective COUPLER}

The schematic structure diagram of the MSC to generate LP11 mode is shown in Fig. 1. The working principle of MSC is coupled-mode mechanism of appeasing the phase-match condition in the fused fibers The MSC was fabricated using weak fusion technique, in order to maintain the geometry of the two fiber and ensures accurate modal conversion efficien y. During

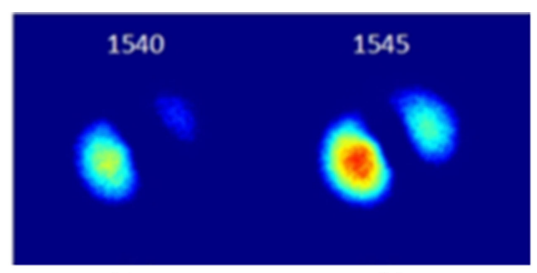

(a)

(b)

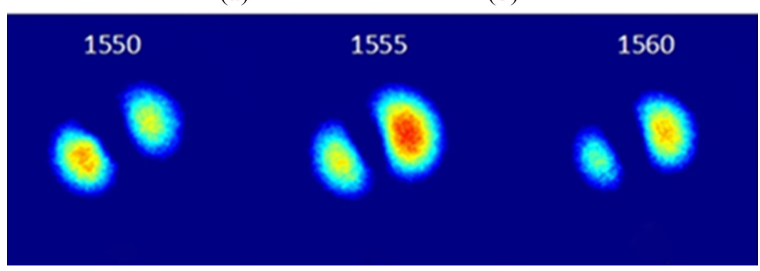

(c)

(d)

(e)

Fig. 2. CCD images of the LP11 mode excited in the TMF at different launching wavelengths.

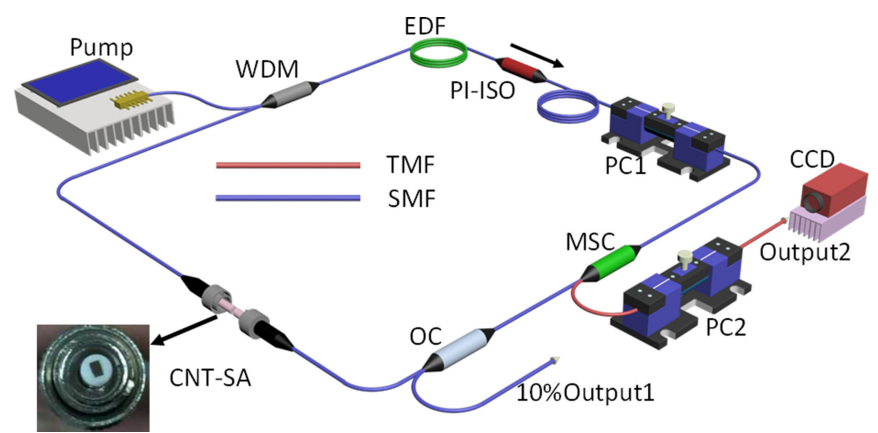

Fig. 3. The experimental setup of the proposed mode-locked fibe laser with CVB generation based on CNTs saturable absorber.

the fabrication process of MSC, the SMF was firstl pre-tapered to about $79 \mu \mathrm{m}$ according to our simulations [26], which was required to meet phase match condition between the LP01 mode in SMF and the LP11 mode in the TMF. Then the TMF was carefully aligned with the pre-tapered SMF and fused together using the modifie flam brushing technique. With tunable laser source launched into the SMF input, the powers of both SMF and TMF output ports were monitored simultaneously by power meter. The two-lobe-shaped intensity patterns at the TMF output port at different wavelengths were also recorded by a CCD (CinCam IR), as shown in Fig. 2. The fabricated MSC can convert LP01 mode efficientl to LP11 mode with a low total insertion loss of about $0.65 \mathrm{~dB}$.

\section{MODE-LOCKED CVB FIBER LASER AND Results}

The experimental setup of the proposed mode-locked fibe laser based on CNTs with CVB generation is shown in Fig. 3. The oscillator has a ring cavity that consists of a $5.4 \mathrm{~m}$ erbium-doped fibe (EDF) with a dispersion parameter $D$ of $-16 \mathrm{ps} / \mathrm{nm} / \mathrm{km}$ at $1550 \mathrm{~nm}$ and a $15.6 \mathrm{~m} \mathrm{SMF}$ with a dispersion parameter $D$ of $17 \mathrm{ps} / \mathrm{nm} / \mathrm{km}$, respectively. The total cavity length is $21 \mathrm{~m}$ and the net dispersion is $-0.227 \mathrm{ps}^{2}$. A polarization independent isolator (PI-ISO) is inserted into the 


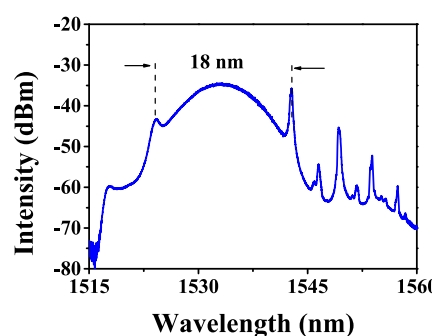

(a)

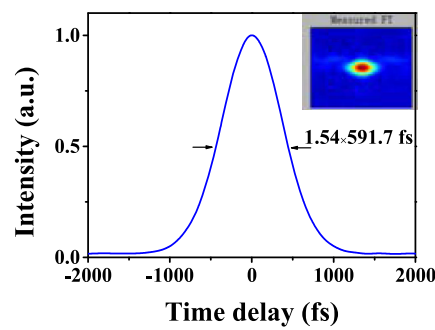

(c)

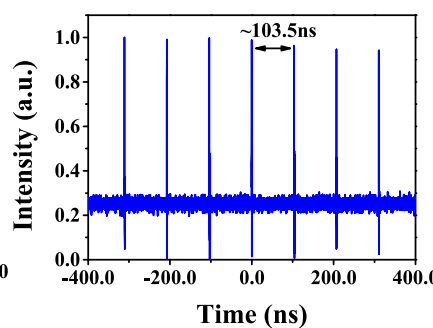

(b)

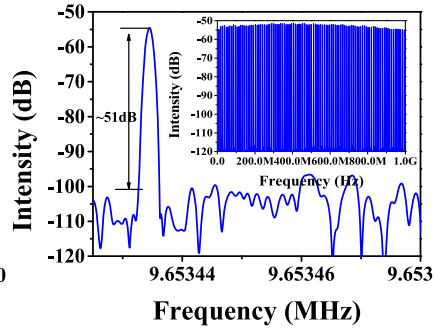

(d)
Fig. 4. (a) Optical spectrum, (b) oscilloscope trace, (c) autocorrelation trace, and (d) RF spectrum of single-wavelength mode-locking state at $1532.5 \mathrm{~nm}$ (The inset of Fig. 4(c) is the measured FROG trace, and the inset of Fig. 4(d) is $\mathrm{RF}$ spectrum with span range of $1 \mathrm{GHz}$ ).

cavity to ensure that light travels unidirectionally in the cavity. The PC1 is used to adjust the polarization state of light in the cavity. The MSC is incorporated into the cavity as a mode converter. Through adjusting the orientations of the extracavity $\mathrm{PC} 2$, both radially and azimuthally polarized beams can be obtained from the output 2 of the MSC. There is no doubt that the PC2 has no influenc on the light in the laser cavity, because PC2 is mounted outside the laser cavity. The fibe laser is pumped by the $980 \mathrm{~nm}$ laser diode with the maximum pump power of $700 \mathrm{mw}$ through a 980/1550 $\mathrm{nm}$ wavelength division multiplexing coupler (WDM). The output 1 is the $10 \%$ port of an optical coupler (OC). The mode-locking mechanism is the saturable absorption of CNTs which is sandwiched between two fibe connectors. The insertion loss and modulation depth of CNTs saturable absorber are around $1.8 \mathrm{~dB}$ and $17 \%$, respectively. The saturable intensity of the CNT-SA is $15.5 \mathrm{MW} / \mathrm{cm}^{2}$. An optical spectrum analyzer (Yokogawa AQ-6370D), a commercial frequency-resolved optical gating (Frog SCAN ultra), a radio-frequency analyzer (FSV30), and a digital storage oscilloscope (LeCroy SDA 6000A) with an electro-photonic detector are used to monitor the laser output 1 simultaneously. The CVB is recorded by the CCD camera (CinCam IR) from the output2 of the MSC.

When the pump power is above the mode-locking threshold value, mode-locking can be easily established. Moreover, depending on the orientation of PC1, various output states: switchable single wavelength and dual-wavelength mode-locking can be obtained.

\section{A. Single-Wavelength Mode-Locking State}

Through adjusting the PC1, mode-locking operation with central wavelength of $1532.5 \mathrm{~nm}$, as shown in Fig. 4(a), can readily be obtained with output power of around $0.6 \mathrm{~mW}$ with the pump power of about $75 \mathrm{~mW}$. The $3-\mathrm{dB}$ spectral width is $5 \mathrm{~nm}$. The evident Kelly sidebands due to the intracavity periodical perturbations are the typical feature of the conventional solitons formed in fibe lasers with anomalous dispersion. The corresponding pulse train is shown in Fig. 4(b), with $103.5 \mathrm{~ns}$ alternation between two adjacent pulses. The corresponding pulse repetition rate is around $9.6 \mathrm{MHz}$, which is just the fundamental frequency. A commercial FROG is used to measure the pulse duration at the output1 port. Since the FROG has low sensitivity and requires relatively high input power of about $10 \mathrm{~mW}$, the output pulses have to be amplifie firs before measuring the pulse duration using the FROG. Then the amplifie pulses are compressed by using proper fibe . The measured autocorrelation trace is illustrated in Fig. 4(c). The inset of Fig. 4(c) is the measured FROG trace. The pulse duration is about $591.7 \mathrm{fs}$ and the time-bandwidth product (TBP) is 0.32 , which indicates the conventional solitons from the fibe laser with anomalous dispersion are almost transform-limited, and the amplificatio has negligible influenc on pulse duration. Fig. 4(d) shows the RF spectrum. A signal-to-noise ratio $51 \mathrm{~dB}$ is exhibited. The fundamental repetition rate is $9.653434 \mathrm{MHz}$, which is in agreement with the calculated value with cavity length. The inset of Fig. 4(d) is the RF spectrum without spectral modulation in wide span range (up to $1 \mathrm{GHz}$ ), highlighting the low-amplitude fluctuatio of the laser and indicating good stability of the single-wavelength mode locking at center wavelength of $1532.5 \mathrm{~nm}$.

According to the phase-matching conditions, the wavelength separation $\Delta \lambda$ between the two first-orde sidebands for the solition is expressed by [37], [38]

$$
\Delta \lambda=\frac{\lambda^{2}}{\pi c \tau_{0}} \sqrt{\frac{4 \pi \tau_{0}^{2}}{\left|\beta_{2}\right|}-1 .}
$$

Where $\lambda$ is the center wavelength of $1532.5 \mathrm{~nm}, \tau_{0}$ is the pulse width, $\beta_{2}$ is the group-velocity dispersion parameter, and then the $\Delta \lambda$ is calculated as $18 \mathrm{~nm}$. The calculated result well agrees with the experimental result.

Keeping the pump power unchanged and adjusting the PC1, we found that the central wavelength of the optical spectrum can be shifted from $1532.5 \mathrm{~nm}$ to $1555.5 \mathrm{~nm}$, as shown in Fig. 5 . The pulse train has also a period of $103.5 \mathrm{~ns}$, as expected from the cavity length. The measured pulse duration is about $508.3 \mathrm{fs}$. The TBP is also close to the transform-limited value, indicating the output pulse is also chirp free. The fundamental repetition rate is $9.653268 \mathrm{MHz}$ from RF spectrum shown in Fig. 5(d). The wavelength separation $\Delta \lambda$ between the two first-orde sidebands is about $21 \mathrm{~nm}$ at current case.

\section{B. Dual-Wavelength Mode-Locking State}

By furthermore adjusting the polarization of the PC1 under the same pump power, the dual-wavelength mode-locking state can be obtained. The optical spectrum is shown in the Fig. 6, and the central wavelengths are $1532.2 \mathrm{~nm}$ and $1555.7 \mathrm{~nm}$, respectively. The pulse train has a period of $103.5 \mathrm{~ns}$ between the two adjacent pulses in Fig. 7, which is also in agreement with the fundamental repetition rate. In principle, two pulses 


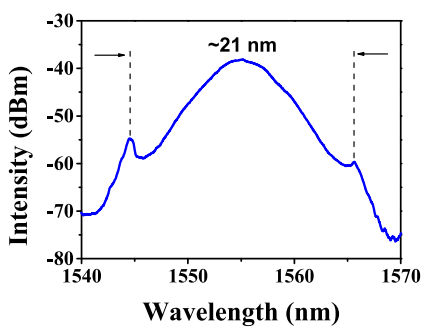

(a)

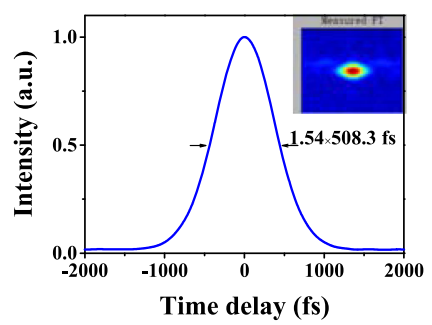

(c)

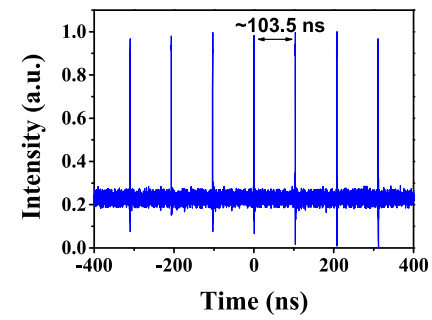

(b)

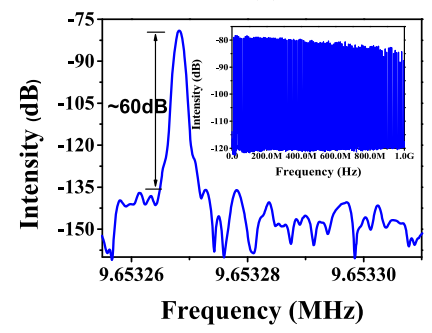

(d)
Fig. 5. (a) Optical spectrum, (b) oscilloscope trace, (c) autocorrelation trace, and (d) RF spectrum of single-wavelength mode-locking state at $1555.5 \mathrm{~nm}$ (The inset of Fig. 5(c) is the measured FROG trace, and the inset of Fig. 5(d) is $\mathrm{RF}$ spectrum with span range of $1 \mathrm{GHz}$ ).

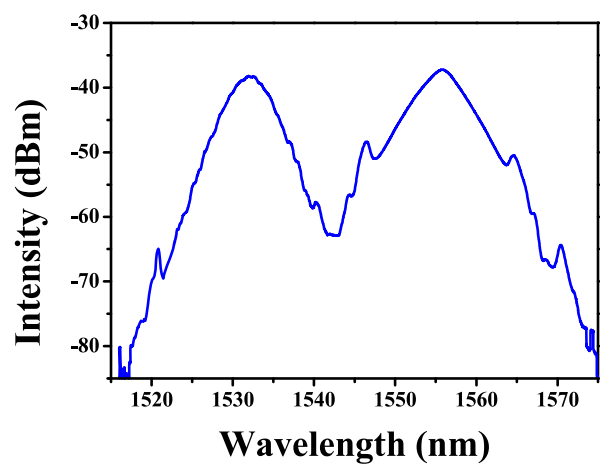

Fig. 6. Optical spectrum of dual-wavelength mode-locking state.

with spectral separation of $\Delta \lambda$ propagating through a segment of fibe with length of $\mathrm{L}$ and dispersion parameter of $\mathrm{D}$, usually results in a group-velocity delay. In current case, the calculated delay is in the order of several picoseconds, which is beyond the detectability of our oscilloscope and photodetector with limited bandwidth (both have $1 \mathrm{GHz}$ bandwidth). Therefore, from the pulse train of dual-wavelength mode-locking, there is only one observed in the oscilloscope. It has been validated that the mode locking really exists at each wavelength of the dual-wavelength mode-locked pulse after filterin one wavelength with a tunable filte .

In order to explore the mechanism of dual-wavelength generation, the MSC was removed from the current laser cavity. Under the condition of maintaining the mode-locked state, no matter how to change the orientations of the PC1, the fibe laser only operates at single wavelength. A typical optical spectrum of mode-locked pulse from the fibe laser with the MSC removed is shown in Fig. 8. It is indicated that the MSC play an important role for dual-wavelength generation. We reckoned that

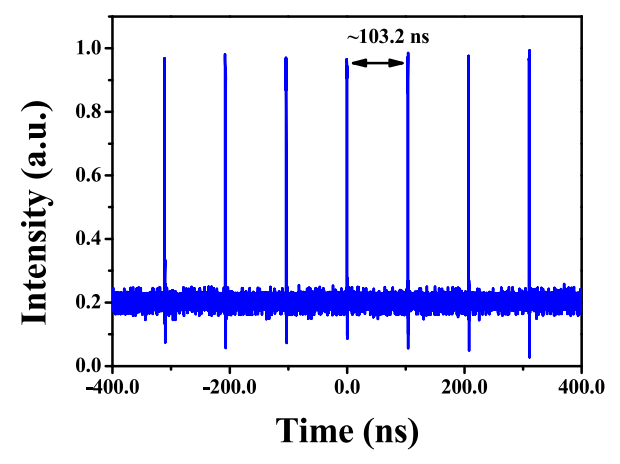

Fig. 7. The pulse train of dual-wavelength mode-locking state.

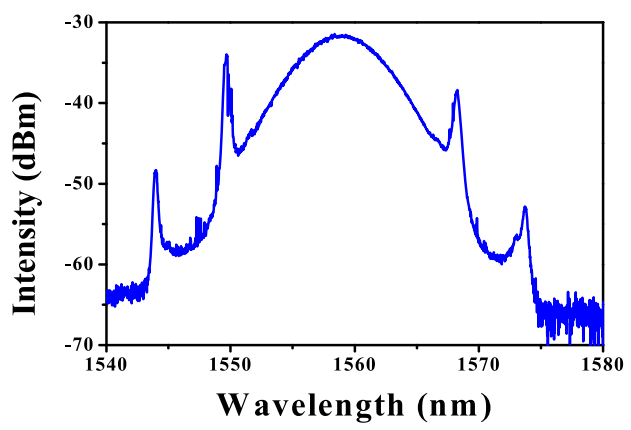

Fig. 8. Optical spectrum of mode-locked pulse from the fibe laser when the MSC was removed.

the dual-wavelength generation mechanism may be the combined result of wavelength-dependent gain distribution of the laser and induced birefringence [39], [40] by the MSC owing to its asymmetrical tapering [41]. Wavelength-dependent intracavity loss has been utilized to implement dual-wavelength generation [42]. The inherent wavelength-dependent gain in a laser can also account for dual-wavelength generation. In consideration of amplifie spontaneous emission (ASE) at $1532 \mathrm{~nm}$ wavelength band and running wavelength of the laser with the MSC at $1555 \mathrm{~nm}$ wavelength band, it is reasonable to assume that the laser has relatively larger gain at these two wavelength bands. If the induced birefringence effect by the MSC leads to wavelength selection with a wavelength spacing of $\sim 23 \mathrm{~nm}$, dual-wavelength operation would become reality.

\section{Intensity Distribution of CVBS}

When the mode-locked fibe laser operates at the different wavelengths, intensity distributions from Output2 have been monitored with CCD camera, for verifying that the CVB can always be generated. After the PC1 in the laser cavity was carefully adjusted to ensure the mode-locking operation and obtain pulsed CVBs, different vector modes with radial and azimuthal polarization states could be reached by controlling the orientations of PC2 to eliminate the degeneracy of the second-order mode from the TMF. The doughnut-shaped intensity profile of both radially and azimuthally polarized beams have been recorded by CCD camera, as shown in Fig. 9(a) and (f). To discriminate radially and azimuthally polarized beams, a linear 

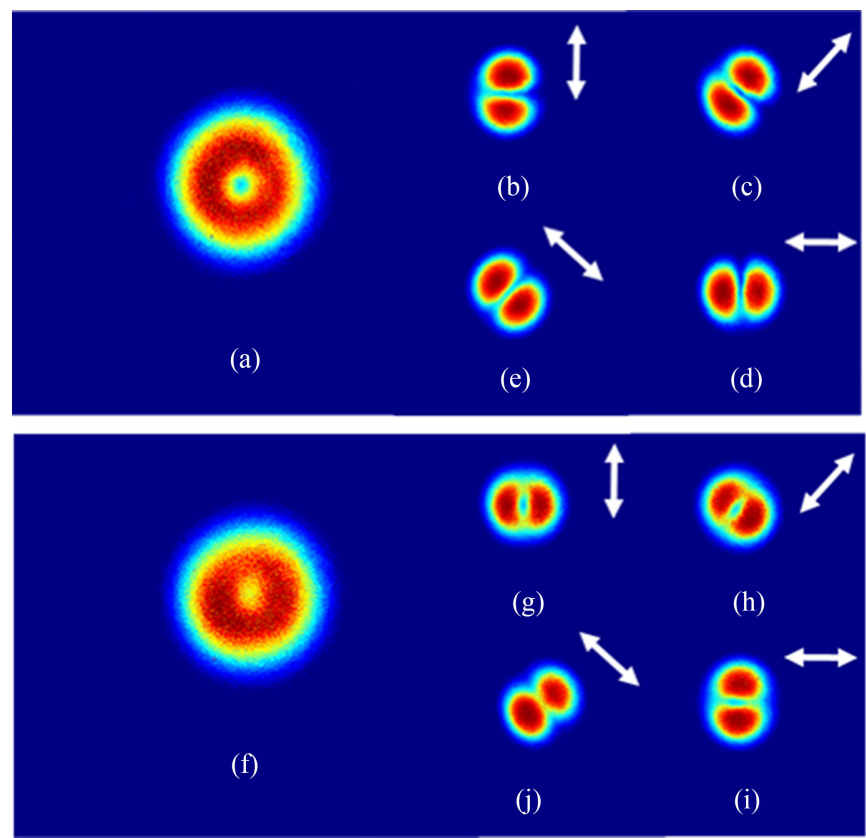

Fig. 9. Intensity distributions of TM01 (a) and TE01 (f) modes and corresponding evolution with rotation of the linear polarizer.

polarizer which can be rotated in a circle, was inserted between the collimator and the CCD camera. The results with arrow indicating the transmission direction of the polarizer are showed in Figs. 9(b)-(e) and (g)-(j), respectively. Two-lobe-shaped intensity patterns are in the same direction as the linear polarizer in Figs. 9(b)-(e), indicating that the generated beam was radially polarized. Contrarily, the directions of the two-lobe-shaped intensity patterns are always perpendicular to the orientation of the polarizer in Figs. 9(g)-(j), which indicates the output laser beam is azimuthally polarized. The purity of both radially and azimuthally polarized beams is estimated to be about $97 \%$ by tight bend approach [15]

It is noted that switchable dual-wavelength CVBs under the operation state of conventional soliton have been obtained in our current fibe laser with net anomalous dispersion. We believe that switchable dual-wavelength CVBs under other operation states, such as stretched pulse, dissipative soliton could also be obtained when the dispersion of the fibe laser is managed appropriately [43]-[45].

\section{CONCLUSION}

In summary, for the firs time a dual-wavelength passively mode-locked cylindrical vector beam fibe laser based on CNTs saturable absorber for mode-locking and MSC for broad mode conversion. Owing to the birefringence filterin effect introduced by the MSC, the CVB fibe laser can operate and switch at two different wavelengths. Moreover, both radially and azimuthally polarized beams can be obtained from the fibe laser. In experiments, the mode-locked fibe laser delivers CVB pulses of dual-wavelength $(1532.5 \mathrm{~nm}$ and $1555.5 \mathrm{~nm})$ and corresponding single wavelength with duration of hundreds of the femtosecond, respectively. This all-fibe -based CVB pulse laser with both wavelength and polarization $\mathrm{fl}$ xibility can provide a cost-effective CVB source for practical applications including mode-division multiplexing optical fibe communication, nanoparticle manipulation, material processing, nonlinear optics, etc.

\section{REFERENCES}

[1] L. S. Yan, X. Liu, and W. Shieh, "Toward the shannon limit of spectral efficien y," IEEE Photon. J., vol. 3, no. 2, pp. 325-330, Apr. 2011.

[2] R. J. Essiambre and R. W. Tkach, "Capacity trends and limits of optical communication networks," Proc. IEEE, vol. 100, no. 5, pp. 1035-1055, May 2012.

[3] B. Goebel, G. J. Foschini, G. Kramer, P. J. Winzer, and R. Essiambre, "Capacity limits of optical fibe networks," J. Lightw. Technol., vol. 28, pp. 662-701, 2010.

[4] A. P. T. Lau, F. Y. M. Chan, and H. Y. Tam, "Mode coupling dynamics and communication strategies for multi-core fibe systems," Opt. Express, vol. 20, pp. 4548-4563, 2012.

[5] Q. Zhan, "Cylindrical vector beams: From mathematical concepts to applications,” J. Syst. Sci. Complexity, vol. 27, pp. 899-910, 2009.

[6] H. Kawauchi, K. Yonezawa, Y. Kozawa, and S. Sato, "Calculation of optical trapping forces on a dielectric sphere in the ray optics regime produced by a radially polarized laser beam," Opt. Lett., vol. 32, pp. 1839 1841, 2007.

[7] L. Novotny, M. R. Beversluis, K. S. Youngworth, and T. G. Brown, "Longitudinal fiel modes probed by single molecules," Phys. Rev. Lett., vol. 86, pp. 5251-5254, 2001.

[8] M. Meier, V. Romano, and T. Feurer, "Material processing with pulsed radially and azimuthally polarized laser radiation," Appl. Phys. A, vol. 86, pp. 329-334, 2007.

[9] D. N. Gupta, N. Kant, E. K. Dong, and H. Suk, "Electron acceleration to GeV energy by a radially polarized laser," Phys. Lett. A, vol. 368, pp. 402-407, 2007.

[10] Z. Y. Rong, Y. J. Han, S. Z. Wang, and C. S. Guo, "Generation of arbitrary vector beams with cascaded liquid crystal spatial light modulators," Opt. Express, vol. 22, pp. 1636-1644, 2014.

[11] X. -L. Wang, J. Ding, W. -J. Ni, C. -S. Guo, and H. -T. Wang, "Generation of arbitrary vector beams with a spatial light modulator and a common path interferometric arrangement," Opt. Lett., vol. 32, pp. 3549-3551, 2007.

[12] S. Ramachandran, P. Kristensen, and M. F. Yan, "Generation and propagation of radially polarized beams in optical fibers" Opt. Lett., vol. 34, pp. 2525-2527, 2009.

[13] D. Lin et al., "Efficient high-power, and radially polarized fibe laser," Opt. Lett., vol. 35, pp. 2290-2292, 2010.

[14] T. Mizunami et al., "Bragg gratings in multimode and few-mode optica fibers" J. Lightw. Technol., vol. 18, pp. 230-235, 2000.

[15] B. Sun et al., "Low-threshold single-wavelength all-fibe laser generating cylindrical vector beams using a few-mode fibe Bragg grating," Opt. Lett., vol. 37, pp. 464-466, 2012.

[16] B. Sun et al., "Transverse mode switchable fibe laser through wavelength tuning," Opt. Lett., vol. 38, pp. 667-669, 2013.

[17] W. Zhang et al., "Cylindrical vector beam generation in fibe with mode selectivity and wavelength tunability over broadband by acoustic fl xural wave," Opt. express, vol. 24, pp. 10376-10384, 2016.

[18] J. Lin et al., "Tungsten disulphide based all fibe Q-switching cylindricalvector beam generation," Appl. Phys. Lett., vol. 107, 2015, Art. no. 191108.

[19] K. Yan et al., "Bi2Te3 based passively Q-switched fibe laser with cylindrical vector beam emission," Appl. Opt., vol. 55, pp. 3026-3029, 2016.

[20] B. Sun et al., "Mode-locked all-fibe laser producing radially polarized rectangular pulses," Opt. Lett., vol. 40, pp. 1691-1694, 2015.

[21] Y. Zhou et al., "Self-starting passively mode-locked all fibe laser based on carbon nanotubes with radially polarized emission," Photon. Res., vol. 4, pp. 327-330, 2016.

[22] D. Mao et al., "Ultrafast all-fibe based cylindrical-vector beam laser," vol. 110, 2017, Art. no. 021107

[23] A. Witkowska, S. Leon-Saval, A. Pham, and T. Birks, "All-fibe LP11 mode convertors," Opt. Lett., vol. 33, pp. 306-308, 2008.

[24] R. Ismaeel, T. Lee, B. Oduro, Y. Jung, and G. Brambilla, "All-fibe fused directional coupler for highly efficien spatial mode conversion," Opt. Expres, vol. 22, pp. 11610-11619, 2014. 
[25] T. Wang et al., "Generation of femtosecond optical vortex beams in allfibe mode-locked fibe laser using mode selective coupler," J. Lightw. Technol., vol. 35, pp. 2161-2166, 2017.

[26] H. Wan et al., "High efficien y mode-locked, cylindrical vector beam fibe laser based on a mode selective coupler," Opt. Express, vol. 25, pp. 11444-11451, 2017.

[27] V. Scardaci et al., "Carbon nanotube polycarbonate composites for ultrafast lasers," Adv. Mater., vol. 20, pp. 4040-4043, 2008.

[28] T. Hasan et al., "Nanotube-polymer composites for ultrafast photonics," Adv. Mater., vol. 21, pp. 3874-3899, 2009.

[29] Z. Sun et al., "Graphene mode-locked ultrafast laser," ACS nano, vol. 4, pp. 803-810, 2010.

[30] J.-S. Lauret et al., "Ultrafast carrier dynamics in single-wall carbon nanotubes," Phys. Rev. Lett., vol. 90, 2003, Art. no. 057404.

[31] D. Brida et al., "Ultrafast collinear scattering and carrier multiplication in graphene," Nature Commun., vol. 4, 2013, Art. no. 1987.

[32] D. Popa et al., "74-fs nanotube-mode-locked f ber laser," Appl. Phys. Lett., vol. 101, 2012, Art. no. 153107.

[33] D. Popa et al., "Sub 200 fs pulse generation from a graphene mode-locked fibe laser," Appl. Phys. Lett., vol. 97, 2010, Art. no. 203106.

[34] L. Nelson, D. Jones, K. Tamura, H. Haus, and E. Ippen, "Ultrashortpulse fibe ring lasers," Appl. Phys. B: Lasers Opt., vol. 65, pp. 277-294 1997.

[35] M. Zhang et al., "Mid-infrared Raman-soliton continuum pumped by a nanotube-mode-locked sub-picosecond Tm-doped MOPFA," Opt. Express, vol. 21, pp. 23261-23271, 2013.

[36] C. Castellani et al., "CW-pumped short pulsed $1.12 \mu \mathrm{m}$ Raman laser using carbon nanotubes," Laser Phys. Letts., vol. 10, pp. 222-228, 2013.

[37] G. P. Agrawal, Nonlinear Fiber Optics. San Francisco, CA, USA: Academic press, 2007

[38] X. Liu, "Soliton formation and evolution in passively-mode-locked lasers with ultralong anomalous-dispersion fibers" Phys. Rev. A, vol. 84, 2011, Art. no. 023835 ,.

[39] Z.-C. Luo et al., "Tunable multiwavelength passively mode-locked fibe ring laser using intracavity birefringence-induced comb filte ," IEEE Photon. J., vol. 2, no. 4, pp. 571-577, Aug. 2010.

[40] Z. Luo et al., "Multiwavelength mode-locked erbium-doped fibe laser based on the interaction of graphene and fibe -taper evanescent field" Laser Phys. Lett., vol. 9, pp. 229-233, 2012.

[41] H. Xuan, J. Ju, and W. Jin, "Highly birefringent optical microfibers" Opt. Express, vol. 18, pp. 3828-39, 2010.

[42] C. Zeng, Y. D. Cui, and J. Guo, "Observation of dual-wavelength solitons and bound states in a nanotube/microfibe mode-locking fibe laser," Opt. Commun., vol. 347, pp. 44-49, 2015.

[43] H. Zhang et al., "Compact graphene mode-locked wavelength-tunable erbium-doped fibe lasers: From all anomalous dispersion to all normal dispersion," Laser Phys. Letts., vol. 7, pp. 591-596, 2010.

[44] X. Liu et al., "Versatile multi-wavelength ultrafast fibe laser mode-locked by carbon nanotubes," Sci. Reports, vol. 3, 2013, Art. no. 2718.

[45] H. Zhang, D. Tang, X. Wu, and L. Zhao, "Multi-wavelength dissipative soliton operation of an erbium-doped fibe laser," Opt. Express, vol. 17, pp. 12692-12697, 2009 .
Zuxing Zhang received the Ph.D. degree from Shanghai Jiao Tong University, Shanghai, China.

He is currently a Professor in the Advanced Photonic Technology Lab, School of Optoelectronic Engineering, Nanjing University of Posts and Telecommunications, Nanjing, China. His research interests include fibe lasers, nonlinear optics, fibe optics, and optical communications.

Yu Cai received the B.S. degree from Nanjing University of Posts and Telecommunications, Nanjing, China.

He is currently working toward the M.S. degree in condensed matter physics with Advanced Photonic Technology Lab, School of Optoelectronic Engineering, Nanjing University of Posts and Telecommunications, Nanjing, China. His research interests include fibe lasers and two-dimensional materials.

Jie Wang received the B.S. degree from Chaohu University, Chaohu, China.

$\mathrm{He}$ is currently working toward the M.S. degree in condensed matter physics with Advanced Photonic Technology Lab, School of Optoelectronic Engineering, Nanjing University of Posts and Telecommunications, Nanjing, China. His research interests include fibe lasers and microfibe .

Hongdan Wan received the Ph.D. degree from Southeast University, Nanjing, China.

She is currently a Lecturer in the Advanced Photonic Technology Lab, School of Optoelectronic Engineering, Nanjing University of Posts and Telecommunications, Nanjing, China. His research interests include fibe lasers, nonlinear optics, fibe optics, and fibe sensors.

Lin Zhang received the Ph.D. degree in physics from the University of Sussex, Sussex, U.K., in 1990.

She was a Research Fellow at the University of Sussex, where he was involved in the fiel of passive and active planar waveguide devices and lasers. She joined the Photonics Research Group, Aston University, Birmingham, U.K., in 1994, and was a Professor in the School of Engineering and Applied Science in 2005. She has been involved mainly in the fiel of fibe grating technologies and applications and extensively involved in a number of LINK, Engineering and Physical Sciences Research Council, European Union, Defence Evaluation and Research Agency, European Regional Development Fund, and Science Research Investment Fund funded research programs. She has authored or coauthored more than 400 international standard journal and conference papers and two books. Her research interests include fibe and waveguide optics and devices, interferometric optics, fibe grating devices, optical sensors and sensing technologies, UV and femtosecond inscription techniques and photosensitivity, and biophotonics. 